\title{
Critical roles of molecular dynamics in the superior mechanical properties of isotactic-poly(1-butene) elucidated by solid-state NMR
}

\begin{abstract}
Toshikazu Miyoshi ${ }^{1}$ and $\mathrm{Al} \mathrm{Mamun}{ }^{2}$
Isotactic-poly(1-butene) (iPB1) shows superior mechanical properties after crystal-crystal transitions. Recently, Miyoshi et al. found that crystalline stems in metastable tetragonal crystal perform uniaxial rotational diffusions accompanying side-chain conformational transitions in the fast motional limit (correlation time, $\left\langle\tau_{\mathrm{c}}\right\rangle\left\langle 10^{-7} \mathrm{~s}\right.$; Macromolecules 2010, 43, 39863989.). In this study, molecular dynamics in stable trigonal crystal is investigated by solid-state nuclear magnetic resonance, which indicates that crystalline stems and side-chain conformations are completely fixed up to melting points $\left.\left(\left\langle\tau_{\mathrm{c}}\right\rangle\right\rangle 10 \mathrm{~s}\right)$. In addition, lamellar thickness, $\langle I\rangle$ of iPB1 and a low isotacticity iPB1 (low_iPB1) with $\langle\mathbf{m m m m}\rangle=\mathbf{7 8 \%}$, respectively, were investigated by small-angle $X$-ray scattering. The low_iPB1 sample shows very week supercooling dependence of $\langle I\rangle(\sim 5 \mathrm{~nm})$, whereas iPB1 shows strong supercooling dependence of $\langle I\rangle(10-28 \mathrm{~nm})$. On the basis of molecular dynamics and $\langle I\rangle$ results, molecular dynamics effects on structures and unique mechanical property of iPB1 are discussed.
\end{abstract}

Polymer Journal (2012) 44, 65-71; doi:10.1038/pj.2011.66; published online 10 August 2011

Keywords: crystal-crystal transition; crystallization; isotactic-poly(1-butene); mechanical property; molecular dynamics; solid-state NMR

\section{INTRODUCTION}

Isotactic poly(1-butene) (iPB1) is an industrially significant material because of its outstanding mechanical strength compared with chemically similar polyolefins, such as polyethylene (PE), isotactic-poly (propylene) (iPP) and isotactic-poly(4-methyl-1-pentene) (iP4M1P). ${ }^{1}$ In addition to having four polymorphs (I, I', II and III), ${ }^{2-5} \mathrm{iPB} 1$ shows complex solid-solid transitions. ${ }^{6-10}$ As the samples crystallize from a melt in stationary conditions, a metastable tetragonal crystal of $11_{3}$ helices (form II) is kinetically favored. This form II spontaneously transforms into a stable trigonal crystal of $3_{1}$ helices (form I) at ambient temperature via a solid-solid transition. ${ }^{6-10}$ As a result, the bulk mechanical and physical properties are enhanced. These effects are caused neither by an increased crystallinity of the material, because the crystal fraction does not change during the transition, nor by variations of morphology. ${ }^{11-14}$ Despite many structural investigations of the solid-solid transition of iPB1, ${ }^{6-14}$ the origin of its outstanding mechanical properties is not fully understood.

The molecular dynamics of polymers are highly related to chain packing and available spaces. Solid-state nuclear magnetic resonance (NMR) has been successfully applied to reveal the molecular dynamics of solid polymers in the amorphous and crystalline regions. ${ }^{15-20}$ In the crystalline regions, the molecular dynamics of typical polyolefins such as $\mathrm{PE},{ }^{16} \mathrm{iPP}^{17,18}$ and $\mathrm{iP} 4 \mathrm{M} 1 \mathrm{P}^{19}$ were investigated by two- and one-dimensional exchange NMR, which can directly detect reorientations of magnetic anisotropic interactions that are due to molecular dynamics. ${ }^{15}$ These crystalline polymers commonly display slow, helical jump motions in the crystalline regions, which include discrete jump motions around the chain axes and translations of overall stems by one monomer unit. It was indicated that such overall motions in the crystalline region have important roles in crystallization, surface melting, mechanical properties and drawability. ${ }^{20}$

There are several solid-state NMR works on iPB1. ${ }^{21-27}$ Maring et al. ${ }^{22}$ using ${ }^{1} \mathrm{H}$ second moments, detected reduced mobility of iPB following solid-solid transitions. Beckham et al. ${ }^{24}$ detected overall segmental motions in form II above $T_{\mathrm{g}}=-23{ }^{\circ} \mathrm{C}$. Maring et al. ${ }^{23}$ investigated molecular dynamics in form I using high-resolution ${ }^{13} \mathrm{C}$ NMR. They did not observe any evidence for molecular dynamics in form $\mathrm{I}$ in the mid $10 \mathrm{kHz}$ range up to $90^{\circ} \mathrm{C}$. However, the detailed dynamic geometry and time-kinetic parameters of $\mathrm{PB} 1$ have not yet been reported.

Very recently, Miyoshi et al. ${ }^{25,26}$ revealed using ${ }^{1} \mathrm{H}-{ }^{1} \mathrm{H}$ dipolar patterns that crystalline stems in form II undergo continuous rotational diffusion around the chain axis, concomitant with lateral conformational transitions in the fast motional limit (correlation time, $\left\langle\tau_{\mathrm{c}}\right\rangle<10^{-7} \mathrm{~s}$ ) at $100^{\circ} \mathrm{C}$. These dynamic characters are direct evidences for dynamically, conformationally disordered crystals. ${ }^{28}$

${ }^{1}$ Department of Polymer Science, The University of Akron, Akron, OH, USA and ${ }^{2}$ Research Institute of Nanotechnology, National Institute for Advanced Industrial Science and Technology, Tsukuba, Ibaraki, Japan

Correspondence: Professor T Miyoshi, Department of Polymer Science, The University of Akron, Goodyear Polymer Center 723, Akron, OH 44325-3909, USA. E-mail: miyoshi@uakron.edu

Received 5 April 2011; revised 19 May 2011; accepted 22 May 2011; published online 10 August 2011 
In this work, we will investigate the molecular dynamics of the stable form I of iPB using center band-only detection of exchange $(\text { CODEX })^{29}$ and modified wide-line separation NMR. ${ }^{30-32}$ As a result, it is found that crystalline stems in form I do not undergo any overall or side-chain motions, which reorient the principle axes of chemical shift anisotropy (CSA) up to $T_{\mathrm{m}}$. On the basis of dynamics results in this and previous works, ${ }^{26}$ we will discuss the relationship between chain packing and the molecular dynamics of forms I and II. In addition, we will investigate how chain mobility influences the lamellar thickness, $\langle l\rangle$, of iPB1. Here we will use two samples of iPB1 and low isotactic PB1 (low_iPB1) $(\langle\mathrm{mmmm}\rangle=78 \%)$. Very recently, De Rosa et al. ${ }^{33}$ found that low tacticity $\mathrm{iPB}$ bypasses crystallization into form II and directly crystallizes into the trigonal form (form $I^{\prime}$ ). The two iPB1 samples used in this study display different crystallization mechanisms (through or bypassing form II). Thereby, we demonstrate that the lamellar thickness of iPB1 is highly dependent on the crystalline mobility. Through information about molecular dynamics and the $\langle l\rangle-1 / \Delta T$ data obtained in this study, we conclude that unique crystallization and irreversible solid-solid transitions dominate the outstanding mechanical properties of iPB1.

\section{EXPERIMENTAL PROCEDURE}

\section{Samples}

Two samples of iPB1 and one of iPP were used in this work. iPB1, with an average molecular weight of $M_{\mathrm{w}}=186000$, a polydispersity of $M_{\mathrm{w}} / M_{\mathrm{n}}=3.3$ and an isotacticity of $\langle\mathrm{mmmm}\rangle=92 \%$, was purchased from Polysciences Inc. (Warrington, PA, USA). iPB, with a low isotacticity of $\langle m m m m\rangle=78 \%$ and an average molecular weight of $M_{\mathrm{w}}=280000$, was kindly provided by Idemitsu Ltd (Sodegaura, Japan). The low isotacticity sample will be referred to as low_iPB. The low_iPB1 was used for investigating $\langle l\rangle$. iPP, with an average molecular weight of $M_{\mathrm{w}}=360000$, a polydispersity of $M_{\mathrm{w}} / M_{\mathrm{n}}=3.3$ and an isotacticity of $\langle m m m m\rangle=97 \%$, was purchased from Polyscience Inc.

We did not further purify any of the samples. The samples were melted between two cover glasses on a hot stage. The thickness of each sample was controlled to be $0.2 \mathrm{~mm}$ by inserting a metal spacer between the cover glasses. Each sample was first melted and then crystallized several times to erase the previous thermal history. In a typical experiment, the samples of iPB1 were melted for $5 \mathrm{~min}$ at $150^{\circ} \mathrm{C}$. The samples were then rapidly transferred to another hot stage that was preset to a required crystallization temperature, $T_{\mathrm{c}}$, to allow for isothermal crystallization under nitrogen atmosphere. The sample was completely solidified, and full crystallization was confirmed by polarized optical microscopy. The equilibrium melting temperatures, $T_{\mathrm{m}}^{0}$, of iPB and low $\_$iPB were determined to be $135 \pm 2$ and $90 \pm 1{ }^{\circ} \mathrm{C}$, respectively, in terms of a Hoffman-Weeks plot using $T_{\mathrm{c}}$ and $T_{\mathrm{m}}$ obtained using differential scanning calorimetry (DSC). iPP samples were crystallized at $150^{\circ} \mathrm{C}$.

\section{Nuclear magnetic resonance}

The ${ }^{13} \mathrm{C}$ solid-state NMR experiments were performed using a Bruker Avance 300 spectrometer (Bruker, Rheinstetten, Germany) equipped with a $4 \mathrm{~mm}$ cross polarization magic-angle spinning (CPMAS) NMR probe. The ${ }^{1} \mathrm{H}$ and ${ }^{13} \mathrm{C}$ carrier frequencies were 300.1 and $75.6 \mathrm{MHz}$, respectively. The MAS frequency was set to $4000 \pm 3 \mathrm{~Hz}$. The $90^{\circ}$ pulses for ${ }^{1} \mathrm{H}$ and ${ }^{13} \mathrm{C}$ were $4.5-5.0 \mu \mathrm{s}$. The recycle delay and cross-polarization time were $2 \mathrm{~s}$ and $1 \mathrm{~ms}$, respectively. High-power ${ }^{1} \mathrm{H}$ two-pulse phase modulation decoupling with a field strength of $65 \mathrm{kHz}$ was used over an acquisition time of $80 \mathrm{~ms}$. The chemical shift was referenced to the $\mathrm{CH}$ signal of adamantine (29.5 p.p.m.) as an external reference. A phase-sensitive ${ }^{1} \mathrm{H}-{ }^{13} \mathrm{C}$ two-dimensional wide-line separation NMR spectrum was obtained in time-proportional phase increment (TPPI) mode. The data matrix had 512 points along the $t_{2}$ dimension and 128 points along the $t_{1}$ dimension, with a dwell time of $2 \mu$ s. A short crosspolarization of $50 \mu$ s was used for the polarization transfer step. Continuouswave decoupling with a field strength of $55 \mathrm{kHz}$ was applied in the $t_{1}$ dimension to suppress ${ }^{1} \mathrm{H}-{ }^{13} \mathrm{C}$ heteronuclear interaction. ${ }^{26,31,32}$ The temperature in the sample was carefully calibrated using the temperature dependence of the ${ }^{207} \mathrm{~Pb}$ chemical shift of $\mathrm{Pb}\left(\mathrm{NO}_{3}\right)_{2} \cdot{ }^{34}$
The CODEX experiments use the recoupling of the CSA interaction using $180^{\circ}$ pulse trains in the two evolution periods sandwiching a mixing period, $t_{\text {mix }}{ }^{29}$ The effect is a signal decay due to the dephasing of the magnetization brought about by changes in the orientation-dependent CSA that results from a reorientational dynamic process during $t_{\text {mix }}$. The magnetization evolves during the initial evolution period, $N t_{\mathrm{r}} / 2$ (where $N=2,4,6$ and so on), under the orientation-dependent CSA interaction, which is recoupled by two successive $180^{\circ}$ pulses per MAS rotation period, $t_{\mathrm{r}}$. The magnetization after the first evolution period is stored along the $\mathrm{z}$ direction by a $90^{\circ}$ pulse and does not dephase during $t_{\text {mix }}$ (which must be set to an integer multiple of $t_{\mathrm{r}}$ ). The magnetization evolves again after the $90^{\circ}$ readout pulse during the second evolution period $\left(N t_{\mathrm{r}} / 2\right)$ and is refocused at its end. The second mixing period, $t_{\mathrm{z}}$, serves as a $\mathrm{z}$-filter and permits the cancellation of longitudinal relaxation. In our experiments, $t_{\mathrm{z}}$ was set to one $t_{\mathrm{r}}$. Following the last $90^{\circ}$ pulse, the signal was detected under ${ }^{1} \mathrm{H}$ two pulse phase modulation decoupling. If there is no molecular motion during $t_{\text {mix }}$, the evolutions in the two evolution periods will cancel each other out, and there will be no decay of the signal intensity. If there is molecular motion during $t_{\mathrm{mix}}$, the orientation-dependent frequency before and after $t_{\text {mix }}$ will be different, and the magnetization will not be completely refocused. The resulting dephasing leads to a decay of the signal intensity in the exchange spectrum $(S)$. To remove the $T_{1}$ and spin-spin relaxation $\left(T_{2}\right)$ effects during $t_{\text {mix }}$ and $N t_{\mathrm{r}}$, a reference spectrum is acquired. It can be obtained simply by interchanging $t_{\text {mix }}$ and $t_{\mathrm{z}}$. The signal intensity in the reference spectrum $\left(S_{0}\right)$ is not sensitive to exchange processes but is only dominated by $T_{1}, T_{2}$ and pulse length errors. The motional correlation time and information about the motional geometry can be obtained by plotting the ratio $\left(S / S_{0}\right)$ versus $t_{\text {mix }}$ and $\left(S / S_{0}\right)$ versus $N t_{\mathrm{r}}$, respectively. A more detailed description of the CODEX experiment can be found in deAzevedo et al. ${ }^{29}$ The MAS frequency was $4000 \pm 3 \mathrm{~Hz}$. The ${ }^{1} \mathrm{H}$ radio frequency (rf) field strength for continuouswave decoupling during the ${ }^{13} \mathrm{C} 180^{\circ}$ pulse with a pulse length of $15 \mu$ s was set to $100 \mathrm{kHz}$. All other $\mathrm{rf}$ parameters were the same as for the CPMAS experiments. The reference and exchange measurements were obtained alternatively every 128 transients to suppress drift of the NMR spectrometer. The $T_{1 \rho \mathrm{H}}$ filter was incorporated into the CODEX pulse program for suppression of the amorphous signal contribution to the CODEX results at ambient temperature and at $-10^{\circ} \mathrm{C}$. In total, each spectrum was obtained by accumulating 1024 transients. The total experimental time for the measurement of the mixing time dependence up to $4 \mathrm{~s}$ was approximately $24 \mathrm{~h}$. The total experimental time of a typical $N t_{\mathrm{r}}$ experiment was approximately $12 \mathrm{~h}$.

\section{Small angle $\mathrm{X}$-ray scattering}

Small angle $\mathrm{X}$-ray scattering studies were performed using a $\mathrm{CuK} \alpha$ radiation generated by a Rigaku Ultrax 4153A 172B X-ray diffractometer (Rigaku, Akishima, Japan) and a point-focusing small angle X-ray scattering camera. The camera length used was $740 \mathrm{~mm}$, and the images were recorded using an image plate with an exposure time of $2.5 \mathrm{~h}$. Digitized data were then read from the image plate using the image plate reader. Using image plate, very small changes in small angle X-ray scattering patterns could be observed with a very short exposure time. The corrected pattern of an empty sample holder was subtracted from each pattern. To calculate the long period and the lamellar thickness, a correlation function method by Rigaku R-axis software (Rigaku) was used.

\section{Differential scanning calorimetry}

All of the samples were measured with an updated computer interfaced PerkinElmer DSC-7 (Perkin-Elmer, Waltham, MA, USA). Both temperature and heatflow levels were corrected by standard materials. Measurements of the melting points were performed at a heating rate of $10{ }^{\circ} \mathrm{Cmin}^{-1}$. To prevent thermal degradation, nitrogen gas was circulated around the sample pan.

\section{RESULTS AND DISCUSSION}

\section{Molecular dynamics in form I}

Figure 1 shows ${ }^{13} \mathrm{C}$ CPMAS NMR spectra of iPB1 crystallized at $100^{\circ} \mathrm{C}$, signal assignments and a DSC chart. The $T_{\mathrm{m}}$ of this sample is $130{ }^{\circ} \mathrm{C}$. At ambient temperature, the ${ }^{13} \mathrm{C}$ crystalline signals of form I are very sharp and dominate the spectrum. Very broad signals with 


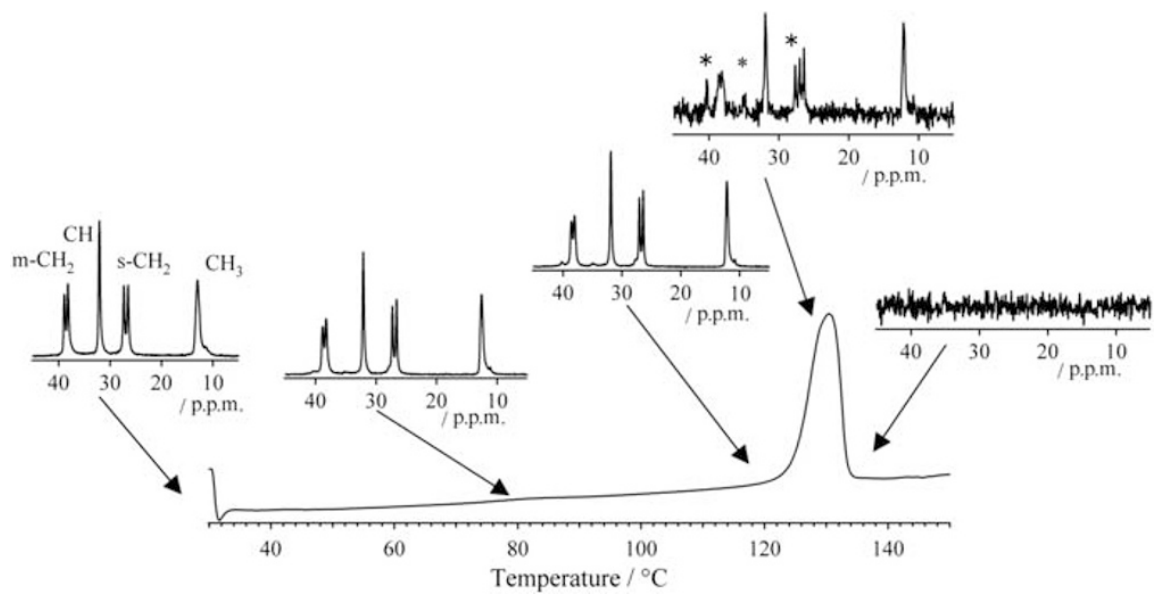

Figure 1 The ${ }^{13} \mathrm{C}$ CPMAS nuclear magnetic resonance spectra of the form I-rich sample of isotactic-poly(1-butene) at ambient temperature, 99, 118, 129 and $136{ }^{\circ} \mathrm{C}$, the signal assignments and the differential scanning calorimetry chart. Asterisks indicate the amorphous signals.

very low intensities appear at the bottom of the sharp crystalline signals. These are amorphous components. There are two reasons for broadening in the amorphous signals: structural disorders such as conformations and packings or a dynamic effect. The $T_{\mathrm{g}}$ of iPB1 is $-23{ }^{\circ} \mathrm{C}$. Thus, the dynamics frequency of thermally activated ${ }^{13} \mathrm{C}$ amorphous segmental motions reaches the mid $10 \mathrm{kHz}$ order at ambient temperature. If molecular dynamics reaches the ${ }^{1} \mathrm{H}$ dipolar decoupling (DD) frequency ( $65 \mathrm{kHz}$ in our experiment), maximum line broadening occurs in the spectrum. This interference effect leads to broadened amorphous signals. The side-chain and main-chain methylene ( $\mathrm{s}-\mathrm{CH}$ and $\mathrm{m}-\mathrm{CH}_{2}$, respectively) carbons in the crystalline regions display doublet signals. Stereoregular polymers can adopt right- or left-handed helical chains. In addition, lateral groups have upward and downward orientations. This orientation disorder provides different atomic positions in $\mathrm{s}-\mathrm{CH}_{2}$ and $\mathrm{m}-\mathrm{CH}_{2}$ carbons and leads to doublet signals (packing effect). ${ }^{2,23}$

At elevated temperature, the ${ }^{13} \mathrm{C}$ line widths of the crystalline signals are almost invariant. Above $99 \pm 2{ }^{\circ} \mathrm{C}$, narrowed amorphous signals (indicated by asterisks) appear in the spectra. At $128 \pm 2{ }^{\circ} \mathrm{C}$, the crystal is mostly melted and only small crystalline signals and further narrowed amorphous signals are observed. At $136 \pm 2{ }^{\circ} \mathrm{C}$, there is no ${ }^{13} \mathrm{C}$ CPMAS signal, indicating complete melting. Through this temperature dependence, ${ }^{13} \mathrm{C}$ line widths of all of the crystalline signals show no broadening up to $T_{\mathrm{m}}$. This result indicates that molecular dynamics in the crystalline region (except for $\mathrm{CH}_{3}$ rotation) do not reach the mid $10 \mathrm{kHz}$ range up to $T_{\mathrm{m}}$.

Figure 2a shows the ${ }^{1} \mathrm{H}$ MAS NMR spectrum for the form I-rich sample of iPB1 at $118^{\circ} \mathrm{C}$. The spectrum is dominated by a very sharp ${ }^{1} \mathrm{H}$ signal corresponding to the amorphous component. The ${ }^{1} \mathrm{H}$ spectrum amplified vertically by a factor of 20 is shown in Figure $2 \mathrm{~b}$. This amplified spectrum clearly shows signals for both the crystalline and the amorphous components. To observe functional ${ }^{1} \mathrm{H}$ line shapes in the crystalline signals, the ${ }^{1} \mathrm{H}-{ }^{13} \mathrm{C}$ wide-line separation spectrum of the form I-rich sample was obtained. Here CP time of $50 \mu$ s was used to suppress the spin-diffusion effect. ${ }^{26,31}$ In addition, ${ }^{13} \mathrm{C}$ DD during the $t_{1}$ detection period was applied to eliminate ${ }^{1} \mathrm{H}-{ }^{13} \mathrm{C}$ heteronuclear dipolar interactions. ${ }^{31,32}$ These two modifications provide short range ${ }^{1} \mathrm{H}-{ }^{1} \mathrm{H}$ dipolar interactions that reflect the local mobility on functional levels. Figures $2 \mathrm{c}$ and $\mathrm{d}$ show ${ }^{1} \mathrm{H}$ slice spectra in the main and side chains, respectively, obtained through the highly resolved ${ }^{13} \mathrm{C}$ signals. The obtained line shapes were fitted using Gaussian functions. The best fitted results indicate that ${ }^{1} \mathrm{H}$ full-line width at half height is
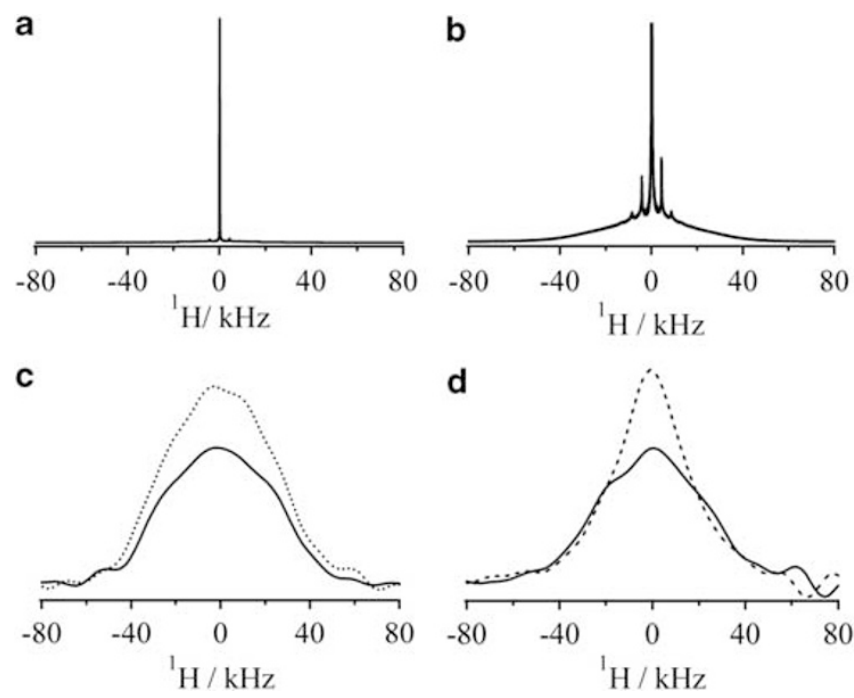

Figure 2 (a) Whole and (b) ${ }^{1} \mathrm{H}$ MAS nuclear magnetic resonance spectrum amplified vertically by a factor of 20 and ${ }^{1} \mathrm{H}$ slice date of ${ }^{1} \mathrm{H}-13 \mathrm{C}$ MAS wideline separation nuclear magnetic resonance spectra of (c) the main chains and (d) the side chains of the form I-rich sample at $118^{\circ} \mathrm{C}$. The dotted lines show the ${ }^{1} \mathrm{H}$ slice date through ${ }^{13} \mathrm{C} \mathrm{CH}$ and $\mathrm{CH}_{3}$ signals, and the solid lines show the ${ }^{1} \mathrm{H}$ ones through ${ }^{13} \mathrm{C} \mathrm{m}-\mathrm{CH}_{2}$ and $\mathrm{s}-\mathrm{CH}_{2}$ signals.

$57,57,54$ and $37 \mathrm{kHz}$, for $\mathrm{m}-\mathrm{CH}_{2}, \mathrm{CH}, \mathrm{s}-\mathrm{CH}_{2}$ and $\mathrm{CH}_{3}$ protons, respectively. The small ${ }^{1} \mathrm{H}$ full-line width at half height for $\mathrm{CH}_{3}$ protons is attributed to fast rotations of the $\mathrm{CH}_{3}$ groups in the crystals. The other ${ }^{1} \mathrm{H}$ line shapes are the typical rigid ones of solid polymers. This means that the overall and side-chain conformations in form I do not undergo large amplitude motions over a ${ }^{1} \mathrm{H}$ line width of $\sim 50 \mathrm{kHz}$ at $118^{\circ} \mathrm{C}$. The small reduction of the ${ }^{1} \mathrm{H}$ line width of s$\mathrm{CH}_{2}$ compared with that of $\mathrm{m}-\mathrm{CH}_{2}$ may be attributed to librational motions of the side chain. This observation is consistent with the former ${ }^{13} \mathrm{C} T_{1}$ reduction of s- $\mathrm{CH}_{2}$ carbon. ${ }^{23}$

To further investigate the slow molecular dynamics in form I crystals, we applied CODEX measurements to iPB1. This method can evaluate slow dynamics in a frequency range of $10^{-1}$ to $10^{3} \mathrm{~Hz}$. This method probes the reorientation of the CSA principle axis directions, which are fixed on ${ }^{13} \mathrm{C}$ atoms due to molecular dynamics. Before investigating the molecular dynamics of iPB1, our pulse 
program was tested using iPP, for which the crystalline stems perform helical jump motions with a jump angle of $120^{\circ}$ at temperatures above $90^{\circ} \mathrm{C} .17,18$ The structure and dynamics are shown in Figure $3 \mathrm{a}$. Several works evaluated helical jump motions using the CODEX method. ${ }^{18,19,29}$

Figure $3 \mathrm{~b}$ shows the $N t_{\mathrm{r}}$ dependence of the CODEX $\left(S / S_{0}\right)$ for all of the functional signals of the $\alpha_{2}$-rich form ${ }^{18}$ of iPP with a $t_{\text {mix }}$ of $200 \mathrm{~ms}$

a
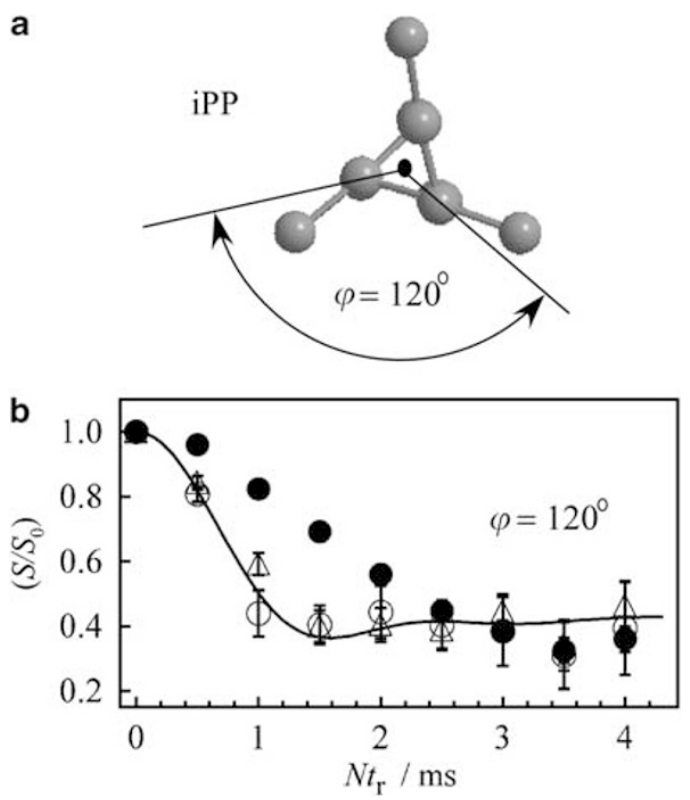

Figure 3 (a) The $3_{1}$ helix of the isotactic-poly(propylene) (iPP) crystalline stem and the jump angle of the $3_{1}$ helix $\left(\varphi=120^{\circ}\right)$. (b) Center bands only detection of exchange $N t_{\mathrm{r}}$ dependence of $\left(S / S_{0}\right)$ for the $\mathrm{CH}_{2}(\mathrm{O}), \mathrm{CH}(\bullet \hat{\imath})$ and $\mathrm{CH}_{3}(\Delta)$ of the isotactic-poly(propylene) $\alpha_{2}$-rich form with $t_{\mathrm{mix}}=200 \mathrm{~ms}$ at $114{ }^{\circ} \mathrm{C}$. The solid curve shows a simulated result for a helical jump with a jump angle of $120^{\circ}$ on $\mathrm{CH}_{2}$ carbon. at $112^{\circ} \mathrm{C}$. The $\left(S / S_{0}\right)$ intensity ratios of all of the signals decay with increasing $N t_{\mathrm{r}}$. This means that all of the functional signals participate in molecular dynamics. The decay curve of each carbon relies on the dynamic geometry, the CSA principle axis values and the orientations. The helical jump effects (a jump angle, $\varphi=120^{\circ}$ ) on $\mathrm{CH}_{2}$ carbon was numerically simulated, and the simulated result is shown as a solid curve in Figure $3 \mathrm{~b}$. Here the CSA size and principle axis orientations reported by Nakai et al. ${ }^{35}$ were used for simulation. This simulation result reproduces the experimental $\left(S / S_{0}\right)$ decay curves well. This confirms that our CODEX pulse program properly works to evaluate molecular dynamics.

Figure 4a shows the CODEX reference $\left(S_{0}\right)$, exchange $(S)$ and difference spectra $\left(S_{0}-S\right)$ of the iPB1 form I with $t_{\text {mix }}=200 \mathrm{~ms}$ and $N t_{\mathrm{r}}=2 \mathrm{~ms}$ at ambient temperature. The result clearly indicates that $S_{0}-S$ is nearly zero in all of the functional signals. The CSA principle axis values of all of the functional signals in $\mathrm{PB}^{36}$ are very similar to other polyolefins. ${ }^{25,35}$ Thus, this result demonstrates that there is no large amplitude motion in iPB1 during $t_{\text {mix }}=200 \mathrm{~ms}$ at ambient temperature. ${ }^{13} \mathrm{C}-{ }^{13} \mathrm{C}$ spin diffusion also leads to an $\left(S / S_{0}\right)$ decay curve at a longer $t_{\text {mix }}$ of more than $1 \mathrm{~s}$ even in natural abundance. Thus, spin diffusion correction is necessary to investigate slow dynamics at any $t_{\mathrm{mix}}$ longer than $1 \mathrm{s.}^{19}$

Figures $4 \mathrm{~b}$ and $\mathrm{c}$ show the $t_{\text {mix }}$ dependence of the $\left(S / S_{0}\right)^{*}$ intensity ratios of $\mathrm{m}-\mathrm{CH}_{2}, \mathrm{CH}$ and $\mathrm{CH}_{3}$ at ambient temperature and at $118^{\circ} \mathrm{C}$, respectively, where asterisk $\left(^{*}\right)$ represents the pure CODEX $\left(S / S_{0}\right)$ decay after spin-diffusion corrections. The spin-diffusion effect, $\left(S / S_{0}\right)_{\mathrm{SD}}$, was obtained at $-10^{\circ} \mathrm{C}$, where no motion is expected. $\left(S / S_{0}\right)^{*}$ is described in terms of $\left(S / S_{0}\right) /\left(S / S_{0}\right)_{\mathrm{SD}}$. The $\left(S / S_{0}\right)^{*}$ intensity ratios of $\mathrm{m}-\mathrm{CH}_{2}$ and $\mathrm{CH}$ carbons do not decay up to $t_{\mathrm{mix}}=4 \mathrm{~s}$ at a fixed $N t_{\mathrm{r}}=2 \mathrm{~ms}$ at ambient temperature. The $\left(S / S_{0}\right)^{*}$ ratios of the $\mathrm{CH}_{3}$ signal at $t_{\text {mix }} \geqslant 2 \mathrm{~s}$ are fluctuated and show large experimental errors. This is caused not by molecular dynamics but by a short ${ }^{13} \mathrm{C} T_{1}$ value. At $118^{\circ} \mathrm{C}$, the $\left(S / S_{0}\right.$ $\left.\left(t_{\text {mix }}\right)\right)^{*}$ intensity ratios of all of the functional signals show similar behaviors to those at ambient temperature. The $t_{\text {mix }}$ dependence of the $\operatorname{CODEX}\left(S / S_{0}\right)$ intensity ratios is analyzed in terms of $\left(S / S_{0}\right)^{*}=$
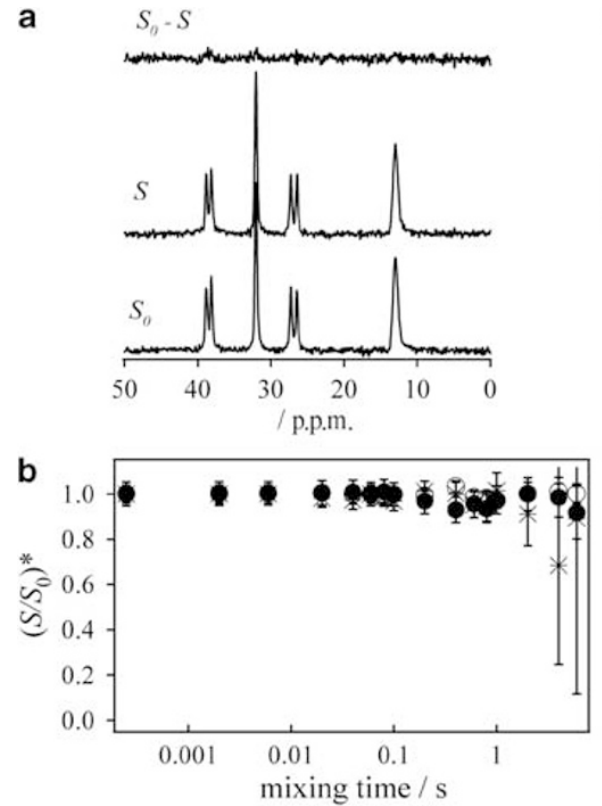

C
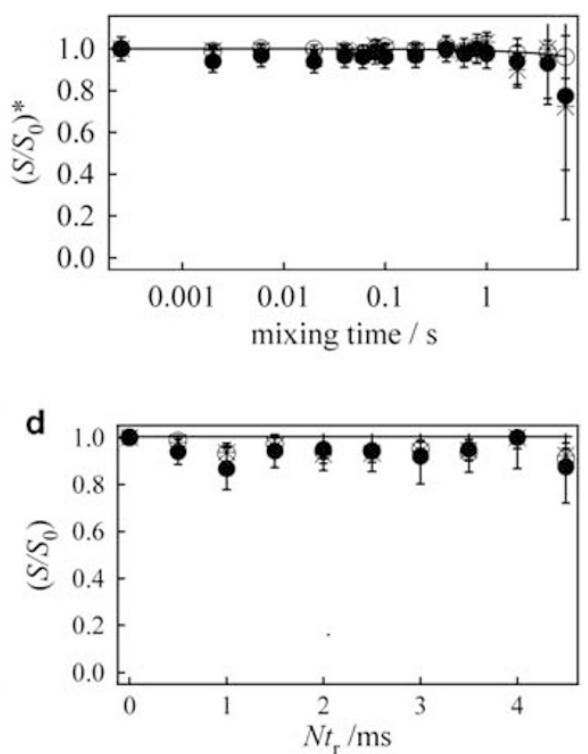

Figure 4 (a) The ${ }^{13} \mathrm{C}$ center bands only detection of exchange reference, exchange and difference spectra for the isotactic-poly(1-butene) form I at a mixing time of $200 \mathrm{~ms}$ and $N t_{r}=2.0 \mathrm{~ms}$ at ambient temperatures. (b, c) Center bands only detection of exchange $t_{\text {mix }}$ dependence of $\left(S / S_{0}\right)^{*}$ and $(\mathbf{d}) N t_{r}$ dependence of the $\left(S / S_{0}\right)$ intensity ratios for $\mathrm{m}-\mathrm{CH}_{2}(\bullet), \mathrm{CH}(\mathrm{O})$ and $\left.\mathrm{CH}_{3}{ }^{*}\right)$ with $N t_{\mathrm{r}}=2 \mathrm{~ms}$ at $(\mathbf{b})$ ambient temperature and at $(\mathbf{c}, \mathbf{d}) 118^{\circ} \mathrm{C}$. 
$1-a\left(1-\exp \left(-\left(t_{\text {mix }} /\left\langle\tau_{\mathrm{c}}\right\rangle\right)^{\beta}\right)\right),{ }^{29}$ where $a$ is related to the available site number, $p$ and $\beta$ is the distribution width. Even at $118^{\circ} \mathrm{C}$, no essential reduction in the $\left(S / S_{0}\right)^{*}$ intensity ratios was observed up to $t_{\text {mix }}=4 \mathrm{~s}$. Figure $4 \mathrm{c}$ shows the best-fit solid curve with $\left\langle\tau_{\mathrm{c}}\right\rangle=860 \pm 5000 \mathrm{~s}$ for the experimental data of $\mathrm{CH}$. This long value is outside of the dynamic window in our experiments $(\leqslant 10 \mathrm{~s})$. In this case, the fitted parameters to the experimental data no longer show physical meaning.

In the $t_{\mathrm{mix}}$ experiment, $N t_{\mathrm{r}}$ was fixed to be $2 \mathrm{~ms}$. This value is a typical condition to characterize large amplitude motions of the crystalline segments. In former works, helical jump motions of iPP and iP4M1P were analyzed using $N t_{\mathrm{r}}=2 \mathrm{~ms} .{ }^{18,19}$ Thus, the current CODEX result denies the presence of large amplitude motions in iPB1 form $\mathrm{I}$ in the slow dynamic range even at very high temperatures just before $T_{\mathrm{m}}$. If iPB1 may have small amplitude motions, the decay curve requires a longer $N t_{\mathrm{r}}$ to detect the molecular dynamics. Figure $4 \mathrm{~d}$ shows the $N t_{\mathrm{r}}$ dependence of the $\left(S / S_{0}\right)$ intensity rations for $\mathrm{m}-\mathrm{CH}_{2}$, $\mathrm{CH}$ and $\mathrm{CH}_{3}{ }^{13} \mathrm{C}$ signals with $t_{\text {mix }}=200 \mathrm{~ms}$ at $118^{\circ} \mathrm{C}$. The $\left(S / S_{0}\right)$ intensity ratios of all of the signals do not decay with increasing $N t_{\mathrm{r}}$ up to $4.5 \mathrm{~ms}$. The solid line in Figure $4 \mathrm{~d}$ represents a jump angle of $0^{\circ}$, which is consistent with the experimental results within the error. This means there is essentially no molecular dynamics in both the main and the side chains in the slow dynamic range. Thus, the dependence of both the $t_{\text {mix }}$ and the $N t_{\mathrm{r}}$ on the CODEX experiments indicates that there are no overall or side-chain motions that induce reorientations of the CSA principle axes of all of the functional signals of form I up to $T_{\mathrm{m}}$. These dynamics results are consistent with the results of ${ }^{1} \mathrm{H}$ line widths and ${ }^{13} \mathrm{C}$ line widths under DD.

Further, the molecular dynamics of form I' was also investigated using the ${ }^{13} \mathrm{C}$ line width under DD and CODEX. The ${ }^{13} \mathrm{C}$ line widths under DD were invariant up to the melting point $\left(78^{\circ} \mathrm{C}\right)$. The CODEX $N t_{\mathrm{r}}$ dependencies of $\mathrm{m}-\mathrm{CH}_{2}, \mathrm{CH}$ and $\mathrm{CH}_{3}$ signals with $t_{\text {mix }}=200 \mathrm{~ms}$ at $75^{\circ} \mathrm{C}$ are shown in Figure 5. None of the signals display a decay up to $N t_{\mathrm{r}}=4.5 \mathrm{~ms}$. The CODEX $t_{\text {mix }}$ dependencies of all of the signals with $N t_{\mathrm{r}}=2 \mathrm{~ms}$ at $75^{\circ} \mathrm{C}$ also indicate no decay of any of the signals up to $t_{\mathrm{mix}}=4 \mathrm{~s}$ (data are not shown). These experimental results indicate that, although there is a significant difference in the melting points between forms I and $\mathrm{I}^{\prime}$, the dynamics characteristics of form $\mathrm{I}^{\prime}$ are very similar to that of form $\mathrm{I}$.

\section{iPB1 versus other crystalline polymers}

Time-kinetic parameters and the geometry of molecular dynamics reflect on their own conformations and chain packing with neighboring molecules (available dynamic space). Our previous results revealed

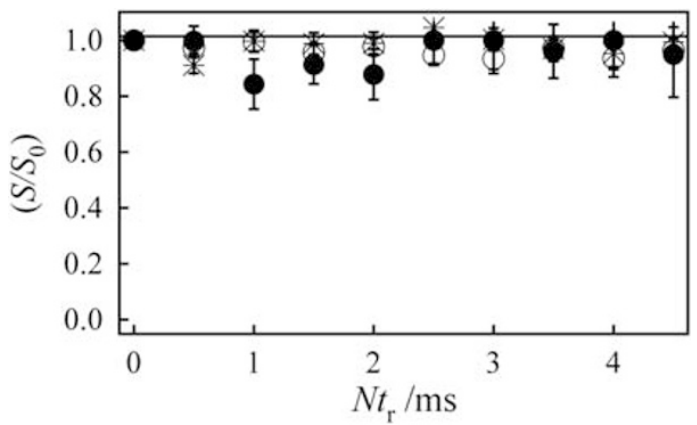

Figure 5 The ${ }^{13} \mathrm{C}$ center bands only detection of exchange reference $N t_{r}$ dependence of the $\left(S / S_{0}\right)$ intensity ratios for $m-\mathrm{CH}_{2}(\bullet), \mathrm{CH}(\mathrm{O})$ and $\mathrm{CH}_{3}$ ${ }^{*}$ ) of low isotacticity isotactic-poly(1-butene) with $t_{\mathrm{mix}}=2 \mathrm{~ms}$ at $75^{\circ} \mathrm{C}$ just before the onset of melting $\left(78^{\circ} \mathrm{C}\right)$. The solid line represents a jump angle of $0^{\circ}$ around the helical axis. that the crystalline stems in form II undergo uniaxial rotational diffusion along with the side-chain conformational transitions in the fast motional limit $\left(\left\langle\tau_{\mathrm{c}}\right\rangle\left\langle 10^{-7} \mathrm{~s}\right)\right.$ at $100^{\circ} \mathrm{C} .{ }^{26}$ Following the subsequent solid-solid transition, the molecular dynamics drastically changes from fast-limit overall dynamics to no overall dynamics on the NMR time scale ( $>10 \mathrm{~s}$ ). This solid-solid transition also accompanies a drastic change in the side-chain dynamics from a fast-limit conformational transition to no transition $(>10 \mathrm{~s})$ even at a temperature close to $T_{\mathrm{m}}$. These drastic mobility changes, both overall and in the side chains, should be simply related to available spaces in the crystalline field. According to crystallographic data, ${ }^{2,3}$ the solid-solid transition leads to $15 \%$ contraction in the $a b$ plane and $12 \%$ extension along the $c$ axis (chain axis). Such densification leads to a huge dynamic gradient between forms II and I.

Here it would be interesting to compare the geometry and the timekinetic parameters of the molecular dynamics observed in forms I and II with those of similar polyolefins. PE (orthorhombic), ${ }^{16}$ iPP $(\alpha 2$ form $)^{17,18}$ and iP4M1P (form I) ${ }^{19}$ commonly show helical jump motions in the crystalline regions. The helical jump motion accompanies jump rotations of overall stems to neighboring sites and translation of overall stems by one monomer unit. Such discrete jump motions are attributed to a well-defined periodicity of chain conformations and packings in the crystalline regions. With regard to the time-kinetic parameters of these dynamics, $\left\langle\tau_{c}\right\rangle$ of the helical jump motions of $\mathrm{PE}, \mathrm{iPP}$ and $\mathrm{iP} 4 \mathrm{M} 1 \mathrm{P}$ were estimated to be $10^{-5}$, $5 \times 10^{-2}$ and $8 \times 10^{-4} \mathrm{~s}$, respectively, at $118^{\circ} \mathrm{C}$ using reported activation energies and correlation times. ${ }^{16,18,19}$ Though the dynamic geometries are different, it is understood that both the overall dynamics in form II $\left(\left\langle\tau_{\mathrm{c}}\right\rangle\left\langle 10^{-7} \mathrm{~s}\right)\right.$ and the absence of overall motions in form $\mathrm{I}\left(\left\langle\tau_{\mathrm{c}}\right\rangle\right.$ $>10 \mathrm{~s}$ ) at $118^{\circ} \mathrm{C}$ are much faster and slower, respectively, than $\left\langle\tau_{\mathrm{c}}\right\rangle=10^{-2}-10^{-5} \mathrm{~s}$ of the helical jump of three polymers at $118^{\circ} \mathrm{C}$. Therefore, it is understood that the dynamic characteristics (geometry and kinetics) of both forms of iPB1 are largely different from those of similar polyolefins.

Similar types of molecular dynamics (geometry and kinetics) to those in form II were observed in other conformationally disordered crystals such as PE in the hexagonal phase under high pressure ${ }^{37}$ and 1,4-trans-polybutadiene in the high temperature phase. ${ }^{38}$ Here chain stems commonly undergo uniaxial rotational diffusions in the fast motional limit. ${ }^{37,38}$ Such unique fast dynamics for PE in the hexagonal packing, 1,4-trans-polybutadiene in the high temperature phase, and form II of iPB are highly related to local structural disorders in both chain packing and conformation.

Compared with dynamic analyses in mobile crystals, there are very few results providing information about immobile polymer dynamics up to the melting point. English and coworkers ${ }^{39}$ clarified that nylon does not show overall stem motions in the crystalline region up to its melting point. Nylon possesses $\mathrm{NH}$ and $\mathrm{CO}$ groups in a repeating unit and shows cooperative hydrogen bonding between stems. In addition, the repeating unit is much longer than in simple polyolefins. These two structural factors highly restrict the overall motions of the stems in nylon. ${ }^{20,39}$ In contrast, iPB1 consists of (i) nonpolar hydrocarbons, (ii) short-length monomer units and (iii) regular helical conformations. These structures are common in similar polyolefins. Thus, only chain packing is a structural factor that restricts both the overall and the side-chain dynamics in form I. In particular, form I of iPB1 has very tight packing structures compared with other polyolefins.

On the basis of molecular dynamics, $\mathrm{Hu}$ and Schmidt-Rohr ${ }^{20}$ categorized semi-crystalline polymers as either mobile crystals or fixed crystals. iPB1 achieves both extremely fast and slow-limit dynamics characteristics through a unique two-step process: 
(i) crystallization into form II and (ii) an irreversible solid-solid transition into form I. We will further investigate how unique molecular dynamics in both forms influence the structures and properties of iPB1.

\section{Mobility effects on lamellar thickness}

Very recently, De Rosa et al..$^{33}$ reported that the low isotacticity sample directly crystallizes into form $\mathrm{I}^{\prime}$ from the melt state. Here the stereoregularity effects on lamellar thickness $\langle l\rangle$ were investigated. Figure 6 a shows the $\langle l\rangle-1 / \Delta T$ relation of two iPB1 samples with different stereoregularity. iPB1 experiences crystallization into form II and a subsequent solid-solid transition into form $\mathrm{I}\langle l\rangle$ was obtained following the solid-solid transition. This sample shows a very wide $\langle l\rangle$ range from $10 \mathrm{~nm}$ at $T_{\mathrm{c}}=50^{\circ} \mathrm{C}$ to $28 \mathrm{~nm}$ at $T_{\mathrm{c}}=110^{\circ} \mathrm{C}$. The best fit to the experimental data provides a slope of $624 \pm 4 \mathrm{~nm} \mathrm{~K}^{-1}$. Low_iPB1 directly crystallizes into form $I^{\prime}$. The $\langle l\rangle-1 / \Delta T$ relation shows a different line with a null slope of $32 \pm 3 \mathrm{~nm} \mathrm{~K}^{-1}$. The $\langle l\rangle$ range is only $4.6-5.3 \mathrm{~nm}$. Therefore, two samples show largely different $1 / \Delta T$ dependencies of $\langle l\rangle$.

Two possible mechanisms might contribute to the largely different slopes in the $\langle l\rangle-1 / \Delta T$ relation for the two samples. One is a purely chemical effect on $\langle l\rangle$. Another is a physical mobility effect in different crystalline forms. There are several works addressing the chemical effects with regard to the lamellar thickness of semi-crystalline polymers. ${ }^{40,41}$ Cheng et al. ${ }^{40}$ investigated the effects of stereoregularity $(\langle m m m m\rangle=78-99 \%)$ on the $\langle l\rangle$ of iPP at various $T_{\mathrm{c}}$ s. We replotted their results in addition to a recent result on the $\langle l\rangle$ of $\mathrm{iPP}^{19}$ over a wide temperature range in Figure $6 \mathrm{~b}$. The low isotacticity samples show thinner lamellar thickness than do those of high isotacticity. Nevertheless, the $\langle l\rangle-1 / \Delta T$ relations of all of the samples are described in terms of one universal line. A similar result was obtained in syndiotactic-poly(propylene-octene) copolymers by Strobl. ${ }^{41}$ These results indicate that chemical disorder influences $\langle l\rangle$ values, but does not change the slope of the universal line of $\langle l\rangle-1 / \Delta T$. Thus, the largely different slopes of the two iPB1 samples must be attributed to a huge difference in mobility between forms I' and II. Extremely fast dynamics in form II leads to large variations in $\langle l\rangle$ at different crystallization temperatures. In contrast, the direct crystallization of form $\mathrm{I}^{\prime}$ into a fixed crystal results in very thin lamellae with $\langle l\rangle=$ approximately $5 \mathrm{~nm}$. This small thickness is reasonably explained in terms of the immobility of the crystalline stems in form I'.
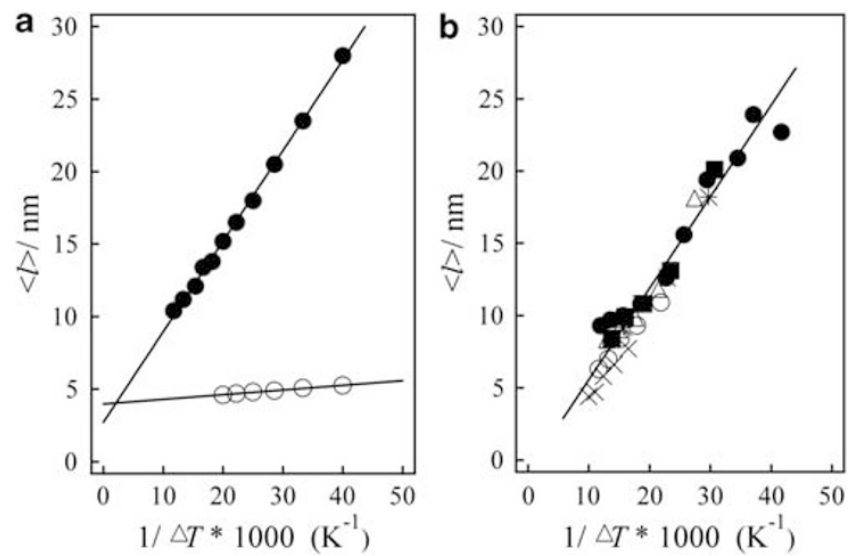

Figure 6 The $\langle I\rangle-1 / \Delta T$ diagram of (a) isotactic-poly(1-butene) with $\langle m m m m\rangle=92(\bullet)$ and $78 \%(\bigcirc)$ and (b) isotactic-poly(propylene) with $\langle m m m m\rangle=99(\mathbf{\square}), 98(\Delta), 97(\bullet), 95\left(^{*}\right), 88(\bigcirc)$ and $78 \%,(\times)$.

\section{Polymorphism}

Here we consider the polymorphism of forms I and I'. So far, several works have focused on the structural difference between forms I and I'. Among the previous characterizations, only DSC detected a large difference in the $T_{\mathrm{m}} \mathrm{s}\left(T_{\mathrm{m}}=130^{\circ} \mathrm{C}\right.$ for form $\mathrm{I}$ and $80-95^{\circ} \mathrm{C}$ for form $\left.\mathrm{I}^{\prime}\right) .^{33,42}$ This result provided direct evidence that form $\mathrm{I}^{\prime}$ and $\mathrm{I}$ are different polymorphs. It was suggested from a large difference in their $T_{\mathrm{m}} \mathrm{s}$ that form $\mathrm{I}^{\prime}$ is structurally disordered form I. ${ }^{42}$ Molecular dynamics is quite sensitive to local environments. If structural disorders are included in form $\mathrm{I}^{\prime}$, significant molecular dynamics might be expected. Our current results, however, did not show any dynamics evidence in form $\mathrm{I}^{\prime}$ up to $T_{\mathrm{m}}$ or in form I. These local dynamics results are consistent with the static structures obtained using XRD, which indicated that both crystalline forms are the same trigonal crystals consisting of $3_{1}$ helices. ${ }^{33}$ These locally static and dynamic structures are not consistent with the structural views of form I' using DSC. As shown above, there is a large difference in the $\langle l\rangle$ values between forms I and I'. The Gibbs-Thomson equation indicates that there is a strong correlation between $T_{\mathrm{m}}$ and $\langle l\rangle$. Thus, a large difference in melting points between forms I and $\mathrm{I}^{\prime}$ is reasonably explained in terms of a huge difference in $\langle l\rangle$ values. Form I is always produced after passing through the extremely mobile crystal, form II. In contrast, form $\mathrm{I}^{\prime}$ is formed by direct crystallization (bypassing form II). Thus, the huge mobility difference between forms I ( $\left.\mathrm{I}^{\prime}\right)$ and II ( $>8$ orders of magnitude) leads to largely different $\langle l\rangle$ and $T_{\mathrm{m}}$ values. In other words, there are different crystallization mechanisms that either pass through or bypass form II and lead to the observed large differences in the $T_{\mathrm{m}}$ and $\langle l\rangle$ values between forms I and I'.

\section{Material properties}

The mechanical properties of bulk samples are affected by crystallinity, lamellae thickness, morphology and chain mobility. Here we explain how (i) a unique crystallization into form II and (ii) the subsequent solid-solid transition into form I results in the superior material properties of iPB1. As discussed above, crystallization into form II leads to very thick lamellae at low supercooling (for example, $\langle l\rangle=28 \mathrm{~nm}$ at $\Delta T=25^{\circ} \mathrm{C}$ ). In contrast, direct crystallization into immobile crystals (form $\mathrm{I}^{\prime}$ ) results in very thin lamellae with $\langle l\rangle=$ approximately $5 \mathrm{~nm}$. This means that immobile crystals cannot produce thick lamellae. The two step process of (i) crystallization into an extremely mobile mesophase and (ii) an irreversible solidsolid transition into the immobile crystal of form I is the only the way to produce very thick lamellae for immobile crystals. Subsequently, storage at ambient temperature induces 'irreversible' solid-solid transitions from the metastable form II into the stable form I. Azzurri et al. reported that forms II-I transitions do not change the lamellar thickness or the crystallinity. ${ }^{13}$ Form I crystals preserve the lamellae thickness and crystallinity that is induced by extremely high chain mobility in form II, depending on the crystallization temperature. As explained above, form I is a fixed crystal, which is largely different from the similar polyolefins $\mathrm{PE},{ }^{16} \mathrm{iPP}^{17,18}$ and $\mathrm{iP}^{\mathrm{M}} 1 \mathrm{P}^{19}$ (mobile crystals). There is a strong correlation between thermally activated molecular dynamics in the crystalline regions and dissipation of the bulk mechanical property. ${ }^{16}$ Thus, $\mathrm{Hu}$ and Schmidt-Rohr ${ }^{20}$ indicated that local overall dynamics in the crystalline regions are the origin of reduction of the bulk mechanical property and the drawability. The absence of molecular dynamics in form I can reasonably explain the superior mechanical properties and low drawability of iPB1 even at very high temperatures. ${ }^{1,43}$ Direct crystallization into form I' also leads to a fixed crystal. However, thin lamellae thickness leads to low melting temperatures. Therefore, it is concluded that only the two- 
step process of (i) unique crystallization and (ii) an irreversible solidsolid transition leads to high crystallinity, thick crystal lamellae and the superior mechanical properties of iPB1.

\section{CONCLUSION}

In this work, it was demonstrated that both forms I and II of iPB show unique molecular dynamics when compared with other semi-crystalline polymers. Form II shows continuous rotational diffusion along with side-chain conformational transitions in the fast motional limit $\left(\left\langle\tau_{\mathrm{c}}\right\rangle<10^{-7} \mathrm{~s}\right)$ at $100^{\circ} \mathrm{C}$. This is direct evidence that form II is a conformationally disordered crystal. Following the irreversible solidsolid transition, the crystalline stems in form I do not undergo any overall or side-chains dynamics up to the melting point in the slow motional limit $\left.\left(\left\langle\tau_{c}\right\rangle\right\rangle 10 \mathrm{~s}\right)$. This immobility was reasonably explained in terms of densifications of the crystalline regions. These huge dynamic contrasts have critical roles for both the structural organization and the material properties of iPB1. Extremely fast dynamics easily leads to very thick lamellae at very high temperatures. Following the solid-solid transition, the immobility of the crystalline stems in thick lamellae is a reason for the maintained bulk mechanical strength at very high temperatures. Comparison of local dynamics in iPB1 with those in similar polyolefins can reasonably explain why only iPB1 shows superior mechanical properties among polyolefins.

1 Gedde, U. W., Viebke, J., Leijström, H. \& Ifwarson, M. Long term properties of hot-water polyolefin pipe-a review. Polym. Eng. Sci. 34, 1773-1787 (1994).

2 Natta, G., Corradini, P. \& Bassi, I. W. Crystal structure of isotactic poly-alpha-butene. Nuov. Chim. Suppl. 15, 52-67 (1960).

3 Turner-Jones, A. Polybutene-1-type II crystalline form. J. Polym. Sci. Part B 1, 455-456 (1963).

4 Petraccone, V., Pirozzi, B., Frasci, A. \& Corradini, P. Polymorphism of isotactic poly- $\alpha-$ butene conformational analysis of the chain and crystalline structure of form 2. Eur. Polym. J. 12, 323-327 (1976).

5 Cojazzi, G., Malta, V., Celotti, G. \& Zannetti, R. Conformational flexibility of isotactic poly-1-olefins. Makromol. Chem. 177, 915-926 (1976).

6 Tuner-Jones, A. Cocrystallization in copolymers of $\alpha$-olefins II-butene- 1 copolymers and polybutene type II/I crystal phase transition. Polymer 7, 23-59 (1966).

7 Gohil, R. M., Milles, M. J. \& Petermann, J. On the molecular mechanism of the crystal transition (tetragonal-hexagonal) in polybutene-1. J. Macromol. Sci. Phys. B21, 189-201 (1982).

8 Nakamura, K., Aoike, T., Usaka, K. \& Kanamoto, T. Phase transformation in poly(1butene) upon drawing. Macromolecules 32, 4975-4982 (1999).

9 Maruyama, M., Sakamoto, Y., Nozaki, K., Yamamoto, T., Kajioka, H., Toda, A. \& Yamada, K. Kinetic study of the II-I phase transition of isotactic polybutene-1. Polymer 51, 5532-5538 (2010).

10 Lotz, B., Mathieu, C., Thierry, A., Lovinger, A. J., De Rosa, C., Ruiz de Ballesteros, O. \& Auriemma, F. Chirality constraints in crystal-crystal transformations: isotactic poly(1butene) versus syndiotactic polypropylene. Macromolecules 31, 9253-9257 (1998).

11 Meng, Y. \& Rieger, J. Synchrotoron ultrasmall-angle X-ray scttering studies on tensile defroamtion of poly(1-butene). Macromolecules 37, 9481-9488 (2004).

12 Tosaka, M., Kamijo, T., Tsuji, M., Kohjiya, S., Ogawa, T., Isoda, S. \& Kobayashi, T. High-resolution transmission electron microscopy of crystal transformation in solution-grown lamellae of isotactic polybutene-1. Macromolecules 33, 9666-9672 (2000).

13 Azzurri, F., Flores, A., Alfonso, G. C., Sics, I. Hsiao, B. S. \& Balta Calleja, F. J. Polymorphism of isotactic polybutene-1 as revealed by microindentation hardness. Part II: correlations to microstructure. Polymer 44, 1641-1645 (2003).

14 Di Lorenzo, M. L., Righetti, M. C. \& Wunderlich, B. Influence of crystal polymorphism on the three-phase structure and on the thermal properties of isotactic poly(1-butene). Macromolecules 42, 9312-9320 (2009).

15 Schmidt-Rohr, K. \& Spiess, H. W. Multidimensional Solid-State NMR and Polymers (Academic Press, London, 1994).

$16 \mathrm{Hu}$, W. G., Boeffel, C. \& Schmidt-Rohr, K. Chain flips in polyethylene crystallites and fibers characterized by dipolar C-13 NMR. Macromolecules 32, 1611-1619 (1999).

17 Schaefer, D., Spiess, H. W., Suter, U. W. \& Fleming, W. W. Two-dimensional solid-state NMR studies of ultraslow chain motion: glass transition in atactic poly(propylene) versus helical jumps in isotactic poly(propylene). Macromolecules 23, 3431-3439 (1990).

18 Miyoshi, T., Mamun, A. \& Hu, W. Molecular ordering and molecular dynamics in isotactic-poly(propylene) characterized by SS-NMR. J. Phys. Chem. B 114, 92-100 (2010).

19 Miyoshi, T., Pascui, O. \& Reichert, D. Slow chain dynamics in isotacticpoly(4-methyl-1-pentene) crystallites near the glass transition temperature characterized by solid-state C-13 MAS exchange NMR. Macromolcules 37, 6460-6471 (2004).

$20 \mathrm{Hu}$, W- G. \& Shimidt-Rohr, K. Polymer ultradrawability: the crucial role of $\alpha$-relaxation chain mobility in the crystallites. Acta. Polym. 50, 271-285 (1999).

21 Belfiore, A. A., Schiling, C. A., Tonelli, A. E., Lovinger, A. J. \& Bovey, F. A. Magic angle spinning carbon-13 NMR spectroscopy of three crystalline forms of isotacticpoly(1-butene). Macromolecules 17, 2561-2565 (1984).

22 Maring, D., Meurer, B. \& Weill, G. ${ }^{1} \mathrm{H}$ NMR Studies of molecular relaxations of poly-1-butene. J. Polym. Sci. Part B 33, 1235-1247 (1995).

23 Maring, D., Whilhelm, M., Spiess, H. W., Meurer, B. \& Weill, G. Dynamics in the crystalline polymorphic forms I and II and III of isotactic poly-1-butene. J. Polym. Sci. Part B 38, 2611-2624 (2000).

24 Beckham, H. W., Schmidt-Rohr, K. \& Spiess, H. W. conformational disorder and its dynamics within the crystalline phase of the form II polymorph of isotactic poly(1-butene). ACS Symp. Ser. 598, 243-253 (1995).

25 Miyoshi, T., Hayashi, S., Imashiro, F. \& Kaito, A. Side-chain conformation and dynamics for the form II polymorph of isotactic poly(1-butene) investigated by highresolution solid-state C-13 NMR spectroscopy. Macromolecules 35, 6060-6063 (2002).

26 Miyoshi, T., Mamun, A. \& Reichert, D. Fast dynamics and conformations of polymer in a conformational disordered crystal characterized by ${ }^{1} \mathrm{H}-{ }^{13} \mathrm{C}$ WISE NMR. Macomoelcules 43, 3986-3989 (2010).

27 Miyoshi, T., Hayashi, S., Imashiro, F. \& Kaito, A. Chain dynamics, conformations, and phase transformations for form III polymorph of isotactic poly(1-butene) investigated by high-resolution solid-state C-13 NMR spectroscopy and molecular mechanics calculations. Macromolecules 35, 2624-2632 (2002).

28 Wunderlich, B., Moller, B., Grebowicz, J. \& Baur, H. conformational motion and disorder in low and high molecular mass crystals. Adv. Polym. Sci. 87, 1 (1988).

29 deAzevedo, E. R., Hu, W. G., Bonagamba, T. J. \& Schmidt-Rohr, K. Centerband-only detection of exchange: efficient analysis of dynamics in solids by NMR. J. Am. Chem. Soc. 121, 8411-8412 (1999).

30 Schmidt-Rohr, K., Clauss, J. \& Spiess, H. W. Correlation of structure, mobility, and morphological information in heterogeneous polymer materials by 2-dimensional wideline-separation NMR-pectroscopy. Macromolecules 25, 3273-3277 (1992).

31 Tekely, P., Palmas, P. \& Mutzenhardt, P. Elimination of heteronuclear dipolar interaction effects from $\mathrm{C}-13$ detected proton spectra in wideline separation nuclear magneticresonance spectroscopy. Macromolecules 26, 7363-7365 (1993).

32 Qiu, X. \& Mirau, A. P. WIM/WISE NMR studies of chain dynamics in solid polymers and blends. J. Magn. Reson. 142, 183-189 (2000).

33 De Rosa, C., Auriemma, F., deBallesteros, O. R., Exposito, F., Laguzza, D., Girolamo, R. D. \& Resconi, L. Crystallization properties and polymorphic behaviors of istactic poly(1-butene) from metallocene catalysts: the crystallization of form I from the melt. Macromolecules 42, 8286-8297 (2009).

34 Takahashi, T., Kawashima, H., Sugisawa, H. \& Baba, T. ${ }^{207} \mathrm{~Pb}$ chemical shift thermometer at high temperature for magic angle spinning experiments. Solid State NMR 15, 119-123 (1999).

35 Nakai, T., Ashida, J. \& Terao, T. Determination of the ${ }^{13} \mathrm{C}$ chemical shift tensors in isotactic polypropylene via the two-dimensional powder pattern in rotating solids. Magn. Reson. Chem. 27, 666-668 (1989).

36 Liu, S. F., Mao, J. D. \& Schmidt-Rohr, K. A robust technique for two-dimensional separation of undistorted chemical-shift anisotropy powder patterns in magic-anglespinning NMR. J. Magn. Reson. 155, 15-28 (2002).

37 de Langen, M. \& Prins, K. O. Mobility of polyethylene chains in the orthorhombic and hexagonal phases investigated by NMR. Chem. Phys. Lett. 299, 195-200 (1999).

38 Möller, M Structure and dynamics of the high temperature polymorph of trans-1,4polybutadiene. Macromol. Chem. Rapid Commun. 9, 107-114 (1988).

39 Hirschinger, J., Miura, H., Gardner, K. H. \& English, A. D. Segmental dynamics in the crystalline phase of nylon 66: solid state deuterium NMR. Macromolecules 23, 2153-2169 (1990).

40 Cheng, S. Z. D., Janimak, J. J., Zhang, A. \& Hsieh, E. T. Isotacticity effect on crystallization and melting in polypropylene fractions: 1 . Crystalline structures and thermodynamic property changes. Polymer 32, 648-655 (1991).

41 Strobl, G. Colloquium: laws controlling crystallization and melting in bulk polymers. Rev. Mod. Phys. 81, 1287-1330 (2009).

42 Boor, J. Jr \& Youngman, E. A. Polymorphism in poly(1-butene): apparent direct formation of modification I. J. Polym. Sci. Patr B 2, 903-907 (1964).

43 Sawai, D., Anyashiki, T., Nakamura, K., Hisada, N., Ono, T. \& Kanaoto, T. Ultradrawing above the static melting temperature of ultra-high molecular weight isotactic poly (1-butene) having low ductility in the crystalline state. Polymer 48, 363-370 (2007). 